\title{
Characterization of the Physical and Mechanical Properties of Femoral Bone Defects Filled with Polyanionic Collagen Scaffolds in Ovariectomized Rats
}

\author{
Marcelo Rodrigues Cunha ${ }^{\mathrm{a}, \mathrm{b}}$, Arnaldo Rodrigues Santos Jr. ${ }^{\mathrm{c} *}$, Leandro Petinari, Gilberto Goissis ${ }^{\mathrm{d}}$, \\ Keico Okino Nonaka ${ }^{\mathrm{e}}$, Charles Chenwei Wang ${ }^{\mathrm{e}}$,Selma Candelária Genari ${ }^{\mathrm{a}, \mathrm{f}}$ \\ ${ }^{a}$ Departamento de Biologia Celular, Instituto de Biologia, \\ Universidade Estadual de Campinas, Campinas, SP, Brasil \\ ${ }^{\mathrm{b}}$ Departamento de Morfologia e Patologia, \\ Faculdade de Medicina de Jundiaí, Jundiaí, SP, Brasil \\ ${ }^{\mathrm{C}}$ Centro de Ciências Naturais e Humanas, \\ Universidade Federal do ABC, Santo André, SP, Brasil \\ ${ }^{\mathrm{d} B i o t e c h ~ B i o m e ́ d i c a ~ P r o d u t o s ~ M e ́ d i c o s ~ e ~ O d o n t o l o ́ g i c o ~ L t d a ~ M E, ~}$ \\ São Carlos, SP, Brasil \\ 'Departamento de Ciências Fisiológicas, \\ Universidade Federal de São Carlos, São Carlos, SP, Brasil \\ ${ }^{\mathrm{f}}$ Centro Estadual de Educação Tecnológica Paula Souza, \\ Faculdade de Tecnologia de Bauru, Bauru, SP, Brasil
}

Received: January 9, 2010; Revised: May 13, 2010

\begin{abstract}
The aim of this study was to evaluate the effect of scaffolds native or polyanionic collagen matrix (submitted to alkaline treatment for 48 or 96 hours, PCM48 or PCM96, respectively) on the repair of osteoporosis bone fractures resulting from the gonadal hormone alterations caused by ovariectomy in rats undergoing hormone replacement therapy. The physical and mechanical characteristics of bone were analyzed. Macroscopic analysis revealed the absence of pathological alterations in the implanted areas. The percent mineral matter and bone mineral density of the femurs were lower in ovariectomized rats. The mechanical strength of newly formed bone was greater in the area receiving the PCM96 scaffolds compared to the area implanted with the native scaffolds. The PCM96 scaffold is the best choice for bone repair in animals with hormone deficiency since it promotes faster bone growth and good mechanical strength.
\end{abstract}

Keywords: bone healing, hormone deficiency, osteoporosis, ovariectomy, polyanionic collagen

\section{Introduction}

The ovary is an endocrine gland responsible for the production of estrogen and progesterone. A decrease in estrogen secretion is observed in cases of early menopause, late menarche ${ }^{1,2}$ and ovariectomy $^{3}$. Deficiency in this hormone results in uncontrolled bone remodeling characterized by a decrease in osteoblastic activity and in bone matrix and reduced deposition of calcium and phosphorus in bone. These alterations can damage bone microarchitecture, predisposing to the occurrence of osteoporosis $^{1,2}$.

Shen et al. ${ }^{3}$ demonstrated that a reduction of estrogen concentration in ovariectomized rats provoked a decrease in bone mineral density and biomechanical changes in the femur, two factors that are important for the prevention of pathological femoral fractures, the most feared event in osteoporosis. Pathological fractures are generally treated surgically by placement of a total prosthesis or by fixation with pins/screws depending on the type and severity of the fractures ${ }^{4}$.

Polyanionic collagen matrices implanted into experimental animals were found to cause no inflammatory response and presented good biocompatibility, and may represent a suitable alternative for bone implants ${ }^{5,6}$. However, studies have shown that the health status of bone is essential for its interaction with biomaterials ${ }^{7}$ and may alter the expected results. Pan et al. $^{8}$ investigated the influence of estrogen deficiency on bone remodeling adjacent to hydroxyapatite implants in the tibia of ovariectomized rats and noted a decrease in both bone volume and implant-bone contact in the recipient area. Thus, osteoporosis might compromise the bone-implant interaction.

One approach to prevent the installation or even to treat osteoporosis is hormone replacement therapy. Although widely used, there are no data in the literature showing the possible effects of osteoporosis or hormone replacement therapy on bone-implant interactions ${ }^{9}$. In view of this fact and of the advantages offered by artificial collagen matrices, the objective of the present study was to evaluate the volume density of newly formed bone at the implant site and the mechanical strength and physical properties of femoral defects filled with polyanionic collagen scaffolds in rats with ovariectomyinduced osteoporosis. 


\section{Materials and Methods}

\subsection{Preparation of the artificial extracellular matrices}

The raw material used for the preparation of the three-dimensional collagen scaffolds was bovine pericardium provided by Braile Biomédica S/A (São José do Rio Preto, SP, Brazil). The samples were prepared and provided by the Institute of Chemistry of São Carlos, University of São Paulo. The following samples were obtained: untreated native three-dimensional collagen matrices (NCM), and polyanionic three-dimensional collagen matrices obtained by alkaline treatment of the collagen matrix for 48 and 96 hours (PCM48 and PCM96, respectively).

\subsection{Animals}

Ninety-six adult female albino Wistar rats (Rattus norvegicus, 12 weeks old), provided by the Multi-Institutional Animal House of the State University of Campinas, were used. The animals were divided into the following groups: group 1, non-ovariectomized animals (NO); group 2, unilaterally ovariectomized animals (UO); group 3, bilaterally ovariectomized animals not submitted to hormone replacement therapy (BOWHRT); group 4, bilaterally ovariectomized animals submitted to hormone replacement therapy (BOHRT). Each group was divided into three subgroups ( $\mathrm{n}=8$ per subgroup) which received the NCM, PCM48 and PCM96 scaffold implants, respectively. The experimental procedure was approved by the Ethics Committee of the institution (CEPex, process 042/07).

\subsection{Ovariectomy and hormone replacement therapy}

The animals were weighed and anesthetized by intramuscular administration of a dose of $0.10 \mathrm{mg} / 100 \mathrm{~g}$ body weight ketamine (Francotar, Sespo Ind, Jacareí, SP, Brazil) and xylazine hydrochloride (2\% Virbaxyl, Virbac Brasil, São Paulo, SP, Brazil) at a proportion of 1:1. A 2-cm incision was made in the skin with a scalpel lateral to the spine to completely remove both ovaries from the pelvic cavity. During the postoperative period the animals received dipyrone in water for 2 weeks. Estradiol hexahydrobenzoate (Benzogynestryl, Hoechst Marion Roussel, French) was used. The drug was diluted in peanut oil (All Chemistry, São Paulo, SP, Brazil) and the animals were injected subcutaneously with $20 \mu \mathrm{g}$ at an average interval of 48 hours from the time of scaffold implantation to the day of sacrifice after 8 weeks.

\subsection{Surgical procedure for the implantation of the native and polyanionic collagen matrices}

The scaffolds were implanted 4 months after ovariectomy. The animals were anesthetized as described above and an incision was made in the skin on the medial side of the thigh, exposing the femoral quadriceps muscle which was sectioned longitudinally in its distal third and separated anterolaterally. With the distal end of the diaphysis of the left femur exposed and the periosteum separated, a bone defect was created with a $5 \mathrm{~mm}$ surgical bur coupled to the pen of a mini-motor. Next, the defect was filled with the artificial matrices. The periosteum was repositioned and closed with No. 6.0 suture. The musculature and skin were closed with No. 4.0 suture.

The animals were sacrificed 8 weeks after implantation of the scaffold into the femur by intramuscular administration of an overdose of the anesthetic (ketamine/xylazine hydrochloride), followed by pneumothorax induced by sectioning of the diaphragm through the abdominal cavity. The femurs with the recipient area were removed, photographed and submitted to mechanical testing and subsequent analysis of their physical properties. Next, the samples were fixed in
Karnovsky's fixative, dehydrated in an increasing ethanol series, cleared in xylene, and embedded in Paraplast. Semi-serial $5 \mu \mathrm{m}$ cross-sections were obtained and stained with hematoxylin and eosin.

\subsection{Morphometric analysis}

Using a square grid with 100 points coupled to the eyepiece of a light microscope, the volume density of newly formed bone at the site of the collagen implant was calculated for each recipient area. Newly formed bone was quantified by stereology according to the Delesse principle cited by Mandarin de Lacerda ${ }^{10}$ using the following formula: $\mathrm{V}_{\mathrm{V}}=\mathrm{P}_{\mathrm{P}} / \mathrm{P}_{\mathrm{T}}(\%)$, where $\mathrm{V}_{\mathrm{V}}=$ volume density or relative volume, $\mathrm{P}_{\mathrm{P}}=$ number of points over newly formed bone, and $\mathrm{P}_{\mathrm{T}}=$ total number of points of the system.

\subsection{Analysis of the biomechanical properties of the femur}

The biomechanical parameters were obtained by compression testing in an Instron 4444 Universal testing machine. A load cell with a maximum capacity of $1 \mathrm{kN}$ was used. Before the test, the bones were thawed at room temperature and kept in saline until the time of the test. The bone was fixed to a support and force was applied perpendicularly to the longitudinal axis of the recipient area in the anteroposterior direction using a cylindric rod with a $3 \mathrm{~mm}$ roller fixed to its end. A pre-load of $5 \mathrm{~N}$ was initially used for accommodation. Next, the load was applied over the recipient area at a constant velocity of $2 \mathrm{~mm} / \mathrm{min}$ until a $2 \mathrm{~mm}$ displacement of the support was observed, and the data were recorded. On the basis of these data, force-deformation curves were constructed for the different tests and the biomechanical parameters were determined using the Matlad software. Stress-strain curves were obtained for the different assays and the mechanical strength of bone can be calculated from these curves ${ }^{11}$.

\subsection{Analysis of the physical properties of the femur}

The femurs were kept in a desiccator for 24 hours, and the immersed weight (IW, weight of bone immersed in distilled water (Archimedes Principle)) and wet weight (WW, wet weight of bone after 24 hours in a desiccator) were then calculated. After this procedure, the bones were dehydrated in an oven at $100{ }^{\circ} \mathrm{C}$ for 24 hours and the dry weight (DW, weight of dehydrated bone after 24 hours at $100{ }^{\circ} \mathrm{C}$ ) was calculated. For the determination of mineral composition, the bones were placed in a muffle furnace at $800{ }^{\circ} \mathrm{C}$ for 24 hours and the mineral weight (MW) was determined. Bone and mineral densities were obtained by calculation of the bone volume according to the Archimedes Principle. The bone percentages of water and mineral matter were also calculated using the following formulas ${ }^{12}$ : bone volume $=\mathrm{WW}-\mathrm{IW}\left(\mathrm{cm}^{3}\right) /$ water density; bone density $=\mathrm{WW} /$ bone volume $\left(\mathrm{g} \cdot \mathrm{cm}^{-3}\right)$; bone mineral density $=\mathrm{MW} /$ bone volume $\left(\mathrm{g} \cdot \mathrm{cm}^{-3}\right)$, and bone percentage of mineral matter $=\mathrm{MW} / \mathrm{WW}(\%)$. On the basis of the results of the biomechanical compression test and bone mineral density, bone quality was calculated using the following formula: $\mathrm{S}=\mathrm{F} \max / \mathrm{BMD}$, where $\mathrm{S}=$ maximum normalized force (bone quality) $\left[\mathrm{N} /\left(\mathrm{mg}_{\mathrm{mm}} \mathrm{m}^{-3}\right)\right] ; \mathrm{F}$ max $=$ maximum load applied to the recipient area until the occurrence of $2 \mathrm{~mm}$ displacement of the support $[\mathrm{N}]$, and $\mathrm{BMD}=$ bone mineral density $\left(\mathrm{mg} \cdot \mathrm{mm}^{-3}\right)$.

\subsection{Statistical analysis}

Linear models were fitted to each of the response variables, with the response being the variable analyzed and the factors the group to which the animals belonged (NO, UO, BOWHRT and BOHRT) and the type of implant (NCM, PCM48 and PCM96). The model was also fitted considering the interaction between group and type of implant. Significance tests were applied to determine the effect of each factor on the response variable. The levels of significance $(p<0.05)$ were adjusted using the Tukey-Kramer test. 


\section{Results}

\subsection{Macroscopic and quantitative analysis}

In NO animals, the NCM and PCM48 implants presented a lower bone interaction in the recipient area than the PCM96 implant (Figure 1a $-a_{3}$ ), as demonstrated macroscopically by the fact that the bone lesion created was still open and the implant was exposed (Figure $1 \mathrm{a}_{1}-\mathrm{a}_{2}$, arrows). In the case of the PCM96 implant, the lesion was partially closed and the implant was covered with periosteum (Figure $1 \mathrm{a}_{3}$, arrow). Similar results

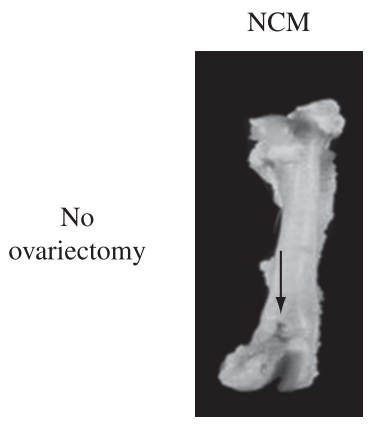

$\left(a_{1}\right)$

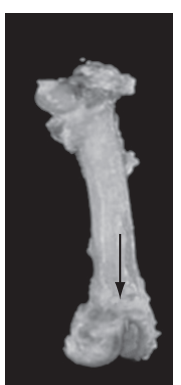

$\left(b_{1}\right)$

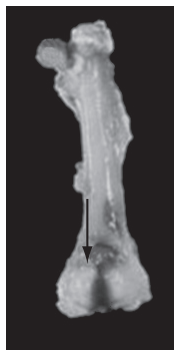

$\left(c_{1}\right)$

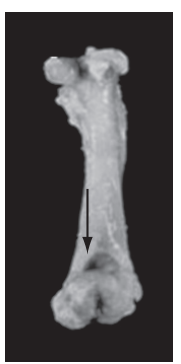

$\left(d_{1}\right)$

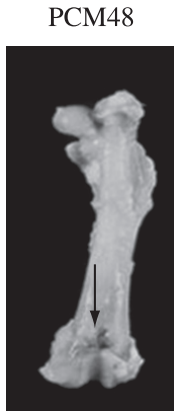

$\left(\mathrm{a}_{2}\right)$

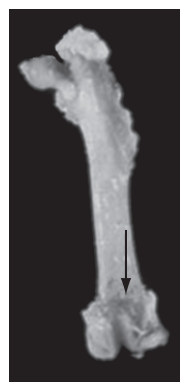

$\left(b_{2}\right)$

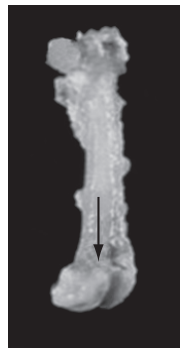

$\left(c_{2}\right)$

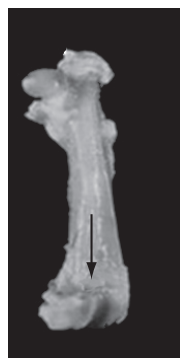

$\left(d_{2}\right)$

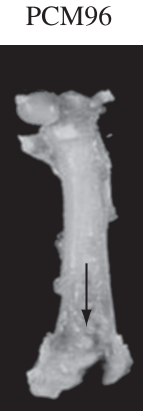

$\left(a_{3}\right)$

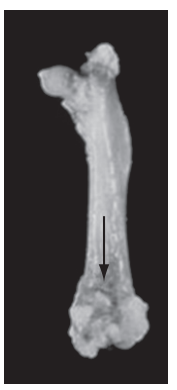

$\left(b_{3}\right)$

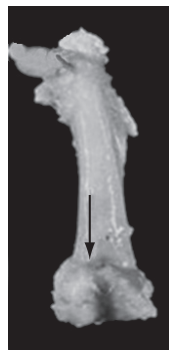

(c)

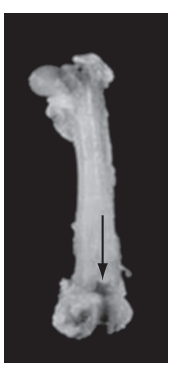

$\left(d_{3}\right)$

\section{$2 \mathrm{~cm}$}

Figure 1. Macroscopic analysis of the implant areas. NCM: native collagen matrix; PCM48: polyanionic collagen matrix submitted to alkaline treatment for 48 hours; PCM96: polyanionic collagen matrix submitted to alkaline treatment for 96 hours.

were observed for UO and BOWHRT animals (Figures $1 b_{1}-b_{3}$ and $1 c_{1}-c_{3}$, respectively). Macroscopic analysis of BOHRT animals showed that, for the three implants studied, the lesions created continued to be partially open and were not covered with periosteum (Figure $1 \mathrm{~d}_{1}-\mathrm{d}_{3}$, arrows). With respect to the quantity of newly formed bone in the implant area, lower bone formation was observed in the BOWHRT group, irrespective of the type of scaffold used. No significant difference in the morphometric data was observed between the NO, UO and BOHRT groups. In addition, the volume of newly formed bone was lower in areas receiving the NCM than in those treated with the polyanionic matrices (Figure 2).

\subsection{Biomechanical properties}

Figure 3 shows the mean maximum strength according to the type of implant (NCM, PCM48, and PCM96) and group (NO, UO, BOHRT, and BOWHRT). Analysis of this variable showed a highly

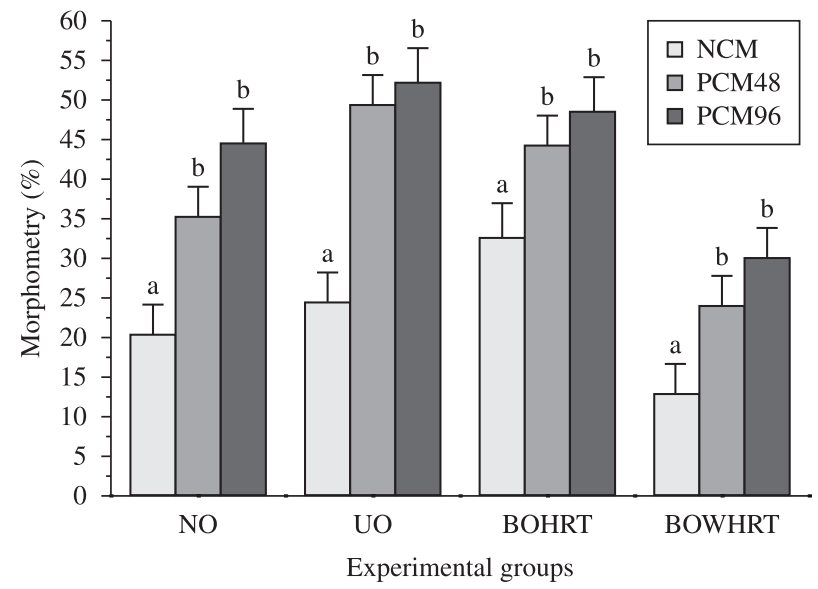

Figure 2. Mean quantity of newly formed bone determined by morphometric analysis. NCM: native collagen matrix; PCM48: polyanionic collagen matrix submitted to alkaline treatment for 48 hours; PCM96: polyanionic collagen matrix submitted to alkaline treatment for 96 hours; NO: non-ovariectomized animals; UO: unilaterally ovariectomized animals; BOWHRT: bilaterally ovariectomized animals not submitted to hormone replacement therapy; BOHRT: bilaterally ovariectomized animals submitted to hormone replacement therapy. $\mathrm{p}<0.05$ indicates statistical significance.

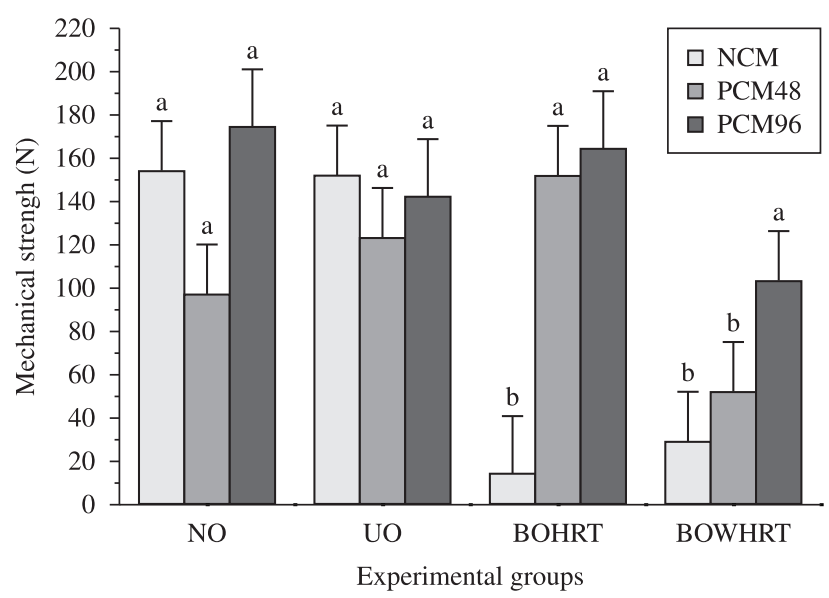

Figure 3. Mean mechanical strength of newly formed bone. NCM: native collagen matrix; PCM48: polyanionic collagen matrix submitted to alkaline treatment for 48 hours; PCM96: polyanionic collagen matrix submitted to alkaline treatment for 96 hours; NO: non-ovariectomized animals; UO: unilaterally ovariectomized animals; BOWHRT: bilaterally ovariectomized animals not submitted to hormone replacement therapy; BOHRT: bilaterally ovariectomized animals submitted to hormone replacement therapy. $\mathrm{p}<0.05$ indicates statistical significance. 


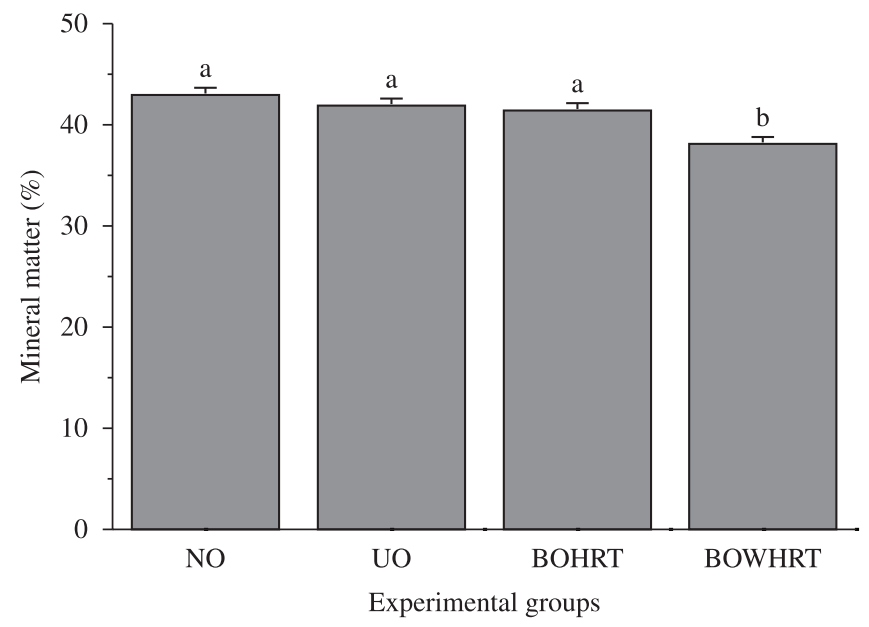

(a)

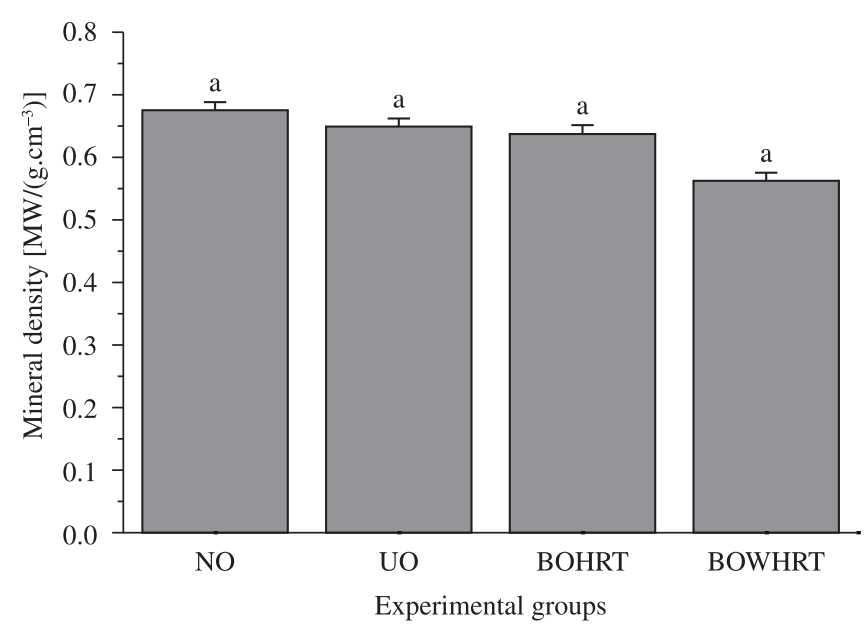

(b)

Figure 4. a) Mean percent mineral matter; and b) mineral density of bone in the different experimental groups. NO: non-ovariectomized animals; UO: unilaterally ovariectomized animals; BOWHRT: bilaterally ovariectomized animals not submitted to hormone replacement therapy; BOHRT: bilaterally ovariectomized animals submitted to hormone replacement therapy. $\mathrm{p}<0.05$ indicates statistical significance.

significant group/implant interaction $(\mathrm{p}<0.05)$. With respect to the $\mathrm{NCM}$, a significant difference was observed between groups, except between the NO and UO groups and between the BOWRHT and BOHRT groups. No significant differences between groups were found for the PCM48 or PCM96. Comparison of the three matrices in each group showed no difference between the native and polyanionic scaffolds in the NO or UO group. In the BOWHRT and BOHRT groups, mechanical strength was greater in the area receiving the PCM96 scaffold compared to the area implanted with the NCM.

\subsection{Physical properties}

Percent mineral matter was lower in the BOWHRT group compared to the other groups (Figure 4a). No significant differences in mineral density were observed between groups (Figure 4b). For the bone quality, similar results occurred between NO and OU and between BOHRT and BOWHRT; however significant differences were found between NO/OU and BOHRT/BOWHRT (Figure 5a). Mean bone density was lower in the BOWHRT group compared to the other groups (Figure $5 b$ ).

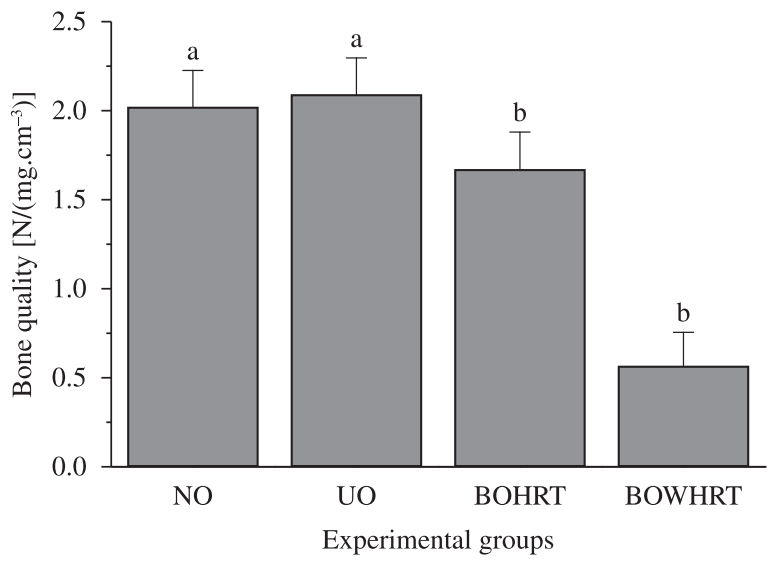

(a)

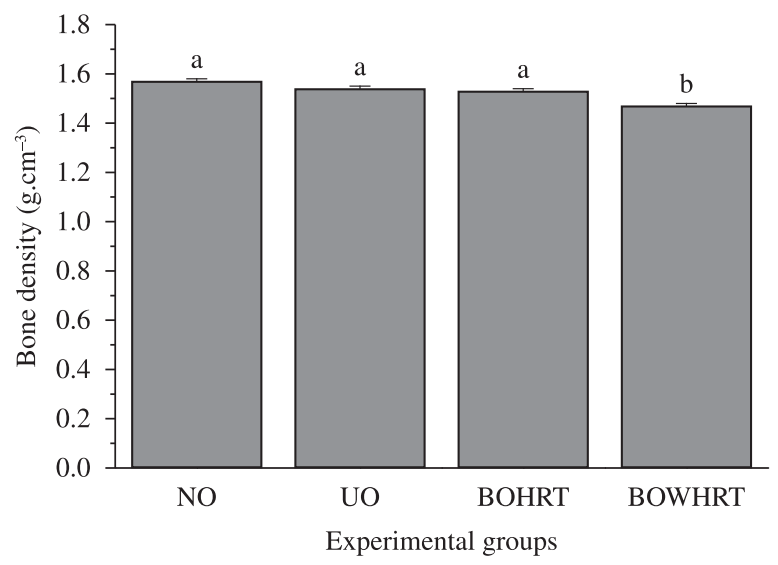

(b)

Figure 5. a) Mean bone quality and; b) mean bone density in the different experimental groups. NO: non-ovariectomized animals; UO: unilaterally ovariectomized animals; BOWHRT: bilaterally ovariectomized animals not submitted to hormone replacement therapy; BOHRT: bilaterally ovariectomized animals submitted to hormone replacement therapy. $\mathrm{p}<0.05$ indicates statistical significance.

\section{Discussion}

Previous studies from our group have shown that osteoblastic cells cultured on polyanionic collagen matrices are able to induce the biomineralization process in vitro ${ }^{13}$ and in vivo ${ }^{5,6}$. In addition, when implanted into nude animals, these polyanionic collagen matrices containing osteoblastic cells induce new bone formation in calvarial bone defects ${ }^{14}$. In view of the highly promising initial results of this material, we decided to study its behavior in a situation in which the recipient bone is of poor quality. In the present experimental model, osteoporotic bone was evaluated, which presents structural and mechanical characteristics distinct from those of healthy bone.

Functionally, the most important mechanical properties of bone are its strength and rigidity. These and other biometric features are best evaluated by analysis of their behavior under loads, i.e., under the influence of externally applied forces ${ }^{9,11}$. This fact becomes important when evaluating the quality of bone and of the synthetic graft material to be used for the treatment of pathological fractures, the most common complications of hormone deficiency-induced osteoporosis. Several studies have reported the deleterious effects of ovariectomy on bone properties, including reduced resistance to external mechanical forces ${ }^{11,15,16,17}$. 
Hypoestrogenemia increases bone resorption and reduces trabecular bone mass, thus affecting the biomechanical strength of bone tissue ${ }^{18}$. This strength depends on bone structure, including the global geometry of bone and the microscopic pattern of the trabecular network ${ }^{19}$. Compared to normal bone, osteoporotic bone is characterized by a reduced number of trabeculae, trabecular thinning, and loss of trabecular connectivity. The overall result is a deterioration of bone strength and an increased fracture susceptibility ${ }^{20}$, however, these authors did not use any type of implant. Ozawa et al. ${ }^{17}$ used titanium implants to treat bone defects created in the femur of ovariectomized rats. Biomechanical tests performed 2 weeks later showed that the mechanical strength of the recipient area in ovariectomized rats was only half the value found in non-ovariectomized animals. In a recent study, Wang et al. ${ }^{21}$ induced an osteoporotic state for the evaluation of $\mathrm{CaSO}_{4}$ resorption and observed that ovariectomized animals exhibited pathological bone changes consistent with the osteoporosis phenotype. The authors suggested that these derangements in bone microarchitecture and physiology accelerated cement resorption and altered the bone response to $\mathrm{CaSO}_{4}^{21}$.

In a previous study, we have shown that the quantity of newly formed bone was significantly lower in animals receiving the NCM compared to PCM48 and PCM96, but no data were obtained regarding the characteristics or quality of the bone formed ${ }^{22}$. In the present study, no difference in mechanical strength was observed between the groups receiving the polyanionic scaffolds despite a higher concentration of newly formed bone in non-ovariectomized animals. The rapid loss of metaphyseal trabecular bone as a result of the imbalance between bone resorption and formation caused by ovariectomy may had a negative influence on the mechanical quality of the recipient area in animals receiving the polyanionic scaffolds.

The mechanical strength of the areas implanted with the native scaffold was higher in intact animals compared to ovariectomized rats not submitted to hormone replacement therapy. However, an increase in bone strength and rigidity does not necessarily indicate improvement in the biomechanical properties of bone tissue ${ }^{23}$. We therefore used a three-point bending biomechanical test. According to Keller and Spengler ${ }^{24}$, in this test bone deformation is due to changes in angular length or shape. This deformation does not exceed 3\% considering the elastic amplitude of the stress-strain curve because bone restores its original shape or length after the load is removed. If load application is continued, the bone tissue reaches its point of deformation followed by rupture, a phase called plastic or non-elastic phase. In the present study, the bone sample contained an implant and we therefore chose to perform a compression test at the implant site. In the three-point bending test, fracture generally occurs in the proximal part, whereas in the present case the implant was located in the distal part. The load for accommodation of the sample should not exceed $10 \%$ of the maximal load; therefore, $5 \mathrm{~N}$ was used which was sufficient to accommodate the sample.

Trabecular architecture is an important factor in osteoporosis and has been quantified by mean of many parameters. However, variations within specimens have been largely ignored. Furthermore, age, disease or drug treatment increases variations in trabecular thickness, which might interfere with the mechanical properties of bone ${ }^{25}$. Comparison of the scaffolds within each group showed no difference in the NO or UO group, whereas a difference was observed in the BOHRT and BOWHRT groups, with the area receiving the PCM96 scaffold presenting greater strength than the area implanted with the native scaffold.

According to Lepola et al. ${ }^{26}$, bone mineral content is one of the determinants of biomechanical bone strength and ovariectomy causes a loss of bone mineral content, especially in trabecular areas ${ }^{27}$.
Katsumata et al. ${ }^{23}$ and Shen et al. ${ }^{3}$ observed a decrease of bone mineral content and density in the femur of ovariectomized rats compared to the intact group, but there was no difference between groups in terms of fracture resistance or rigidity. Wang et al. ${ }^{25}$ evaluated the influence of osteoporosis on the middle and late periods of tibia fracture healing in ovariectomized rats, analyzing histomorphological changes, bone mineral density and biomechanical properties. In that study, bone mineral density and callus failure stress were lower in the ovariectomized group compared to the sham group. In the present study, a decrease in bone mineral density and percent mineral matter was observed in ovariectomized rats not submitted to hormone replacement therapy, but these animals did not differ in terms of mechanical strength of the area receiving the polyanionic scaffolds.

Ovariectomized animals are frequently used as models in the study of postmenopausal osteoporosis; however, some discrepancies exist in the literature regarding bone-implant interaction when biomaterials such as titanium and ceramics are used. For example, some studies reported significant bone loss around these biomaterials, whereas others found no difference in bone growth in the recipient areas of ovariectomized animals ${ }^{17}$. Differences in the results of human studies have also been reported. In a study involving 30 postmenopausal women ranging in age from 46 to 62 years with a diagnosis of osteoporosis and alveolar bone defects, a biphasic calcium phosphate/poly-DL-lactide-co-glycolide (BCP/PLGA) composite was implanted into the osteoporotic alveolar bone. Six weeks after implantation, bone density was lower in the region of osteoporotic bone receiving the implant than in untreated control bone. On the other hand, 24 weeks after implantation bone density was significantly higher when compared to the untreated region ${ }^{28}$.

Analysis of some physical properties of the femurs revealed a significant difference between intact animals and ovariectomized rats not submitted to hormone replacement therapy. Similar results have been reported by Hietala ${ }^{29}$ and Nordsletten et al. ${ }^{16}$ who observed a reduction in femoral bone density and volume in ovariectomized rats. However, in the present study bone volume was higher in ovariectomized animals not submitted to hormone replacement therapy, although wet weight, a variable used to calculate bone volume and density, was similar in the groups. Controversial data regarding the lack of change in wet weight have also been reported in other studies ${ }^{11,16}$

In the present experiment, macroscopic and quantitative analyses showed that the sites receiving the polyanionic collagen matrices (PCM48 and PCM96) presented the best interaction and capacity of bone neoformation when compared to areas implanted with the native collagen scaffold. In addition, in ovariectomized rats bone strength was greater in areas implanted with the PCM96 scaffold.

The present findings are also compatible with previous results from our group showing a significant advantage of PCM96 compared to other scaffolds when implanted into animals with experimentally induced osteoporosis ${ }^{22}$. However, it is not sufficient that these implants induce bone formation. It is also necessary that this newly formed bone is of good quality, i.e., its strength is compatible with that of normal bone. In the present study, bone tissue of satisfactory quality was obtained with the PCM96 matrix in all situations studied. The PCM48 matrix also promoted fairly good mechanical strength, except for animals with experimental osteoporosis.

The present results suggest that polyanionic scaffolds are a promising biomaterial for the regeneration of bone defects due to their qualities. In cases of osteoporosis due to hormone deficiency, the PCM96 scaffold is the best choice for bone repair since it promotes faster bone growth and good mechanical strength. 


\section{References}

1. Rehman HU and Masson EA. Neuroendocrinology of female aging. Gender Medicine. 2005; 2(1):41-56.

2. Rasgon N, Shelton S and Halbreich U. Perimenopausal mental disorders: epidemiology and phenomenology. CNS Spectrums. 2005; 10(10):471-478.

3. Shen V, Birchman R, Wu DD and Lindsay R. Skeletal effects of parathyroid hormone infusion in ovariectomized rats with or without estrogen repletion. Journal of Bone and Mineral Research. 2000; 15(4):740-746.

4. Kanis JA. Consequences of osteoporosis. In Kanis JA, (editor). Textbook of Osteoporosis. London: Blackwell; 1996. p. 200-225.

5. Rosa FP, Lia RCC, Souza KOF, Goissis G and Marcantonio Jr. E. Tissue response to polyanionic collagen:elastin matrices implanted in rat calvaria, Biomaterials. 2003; 24(2):207-212.

6. Rocha LB, Adam RL, Leite NJ and Metze K and Rossi MA. Biomineralization of polyanionic collagen-elastin matrices during calvarial bone repair. Journal of Biomedical Material Research. 2006; 79A(2):237-245.

7. Albrektsson T, Branemark PI, Hansson HA and Lindstrom J. Osseointegrated titanium implants. Acta Orthopaedica Scandinavica. 1981; 52(2):155-170.

8. Pan J, Shirota T, Ohno K and Michi K. Effect of ovariectomy on bone remodeling adjacent to hydroxyapatite-coated implants in the tibia of mature rats. Journal of Oral and Maxillofacial Surgery. 2000; 58(8):877-882.

9. Fini M, Giavaresi G, Torricelli P, Borsari V, Giardino R, Nicolini A et al. Osteoporosis and biomaterial osteointegration. Biomedicine and Pharmacotherapy. 2004; 58(9):487-493.

10. Mandarim de Lacerda CA. What is the interest of normal and pathological morphological research to be quantitative? The example of the stereology. Brazilian Journal of Morphological Science. 1999; 16:131-139.

11. Peng Z, Tuukkanen J, Zhang H, Jamsa T and Vaananen HK. The mechanical strength of bone in different rat models of experimental osteoporosis. Bone. 1994; 15(5):523-532.

12. Martin RB. Effects of simulated weightlessness on bone properties in rats. Journal of Bone and Mineral Metabolism. 1990; 21:91-97.

13. Moreira PL, An YH, Santos Jr AR and Genari SC. In vitro analysis of anionic collagen scaffolds for bone repair. Journal of Biomedical Material Research. 2004; 71B(2):229-237.

14. Cunha MR, Santos Jr AR and Genari SC. Cultura de osteoblastos sobre membranas de colágeno polianiônico: avaliação preliminar do potencial de indução da formação de tecido ósseo visando reparação tecidual. Boletim de Medicina Veterinária. 2005; 1(1):73-85.

15. Fonseca D and Ward WE. Daidzein together with high calcium preserve bone mass and biomechanical strength at multiple sites in ovariectomized mice. Bone. 2004; 35(2):489-497.
16. Nordsletten L, Kaastad TS, Madsen JE, Reikeras O, Ovstebo R, Stromme $\mathrm{JH}$ et al. The development of femoral osteopenic in ovariectomized rats in no reduced by high intensity treadmill training: a mechanical and densitometric study. Calcified Tissue International. 1994; 55(6):436-443.

17. Ozawa S, Ogawa T, Iida K, Sukotjo C, Hasegawa H, Nishimura RD et al. Ovariectomy hinders the early stage of bone-implant integration: histomorphometric, biomechanical, and molecular analyses. Bone. 2002; 30(1):137-143.

18. Ikeda S, Tsurukami H, Ito M, Sakai A., Sakata T, Nishida S et al. Effects of trabecular bone contour on ultimate strength of lumbar vertebra after bilateral ovariectomy in rats. Bone. 2001; 28(6):625-633.

19. Benhamou CL, Lespessailles E and Royant V. Bone structure and mechanical resistance of the bone tissue. Presse Medicale. 1996; 25(6):249-254.

20. Hawker GA. The epidemiology of osteoporosis. Journal of Rheumatology. 1996; 45(9):2-5.

21. Wang ML, Massie J, Perry A, Steven R and Kim CW. A rat osteoporotic spine model for the evaluation of bioresorbable bone cements. Spine Journal. 2007; 7(4):466-474.

22. Cunha MR, Santos Jr. AR, Goissis G and Genari SC. Implants of polyanionic collagen matrix in bone defects of ovariectomized rats. Journal of Materials Science: Materials in Medicine. 2008; 19(3):1341-1348.

23. Katsumata T, Nakamura T, Ohnishi $\mathrm{H}$ and Sakurama T. Intermittent cyclical etidronate treatment maintains the mass, structure and the mechanical property of bone in ovariectomized rats. Journal of Bone and Mineral Research. 1995; 10(6):921-931.

24. Keller TS and Spengler DM. Regulation of bone stress and strain in the immature and mature rat femur. Journal of Biomechanics. 1989; 22(11-12):1115-1127.

25. Wang JW, Li W, Xu SW, Yang DS, Wang Y, Lin M et al. Osteoporosis influences the middle and late periods of trabeculae healing in a rat osteoporotic model. Chinese Journal Traumatology. 2005; 8(2):111-116.

26. Lepola VT, Hannuniemi R, Kippo K, Lauren L, Jalovaara P and Vaananen H.K. Long term effects of clodronate on growing rat bone. Bone. 1996; 18(2):191-196.

27. Westerlind KC, Wronski TJ, Ritman EL, Luo ZP, An KN, Bell NH et al. Estrogen regulates the rate of bone turnover but bone balance in ovariectomized rats is modulated by prevailing mechanical strain. Physiology. 1999; 94:4199-4104.

28. Zorica A, Nenad I, Dragan P and Dragan U. Substitution of osteoporotic alveolar bone by biphasic calcium phosphate/poly-DL-lactideco-glycolide biomaterials. Journal of Biomaterials Applications. 2007; 21(3):317-328.

29. Hietala EL. The effect of ovariectomy on periosteal bone formation and bone resorption in adult rats. Bone and Mineral. 1993; 20(1):57-65. 